\title{
Assessment of Soil Microbial Status under Different Land Use Systems in North Western Zone of Kashmir
}

\author{
Fozia Shafiq Wani ${ }^{1}$, Farida Akhter ${ }^{1}$, Shakeel Mir ${ }^{1}$, Zahoor Ahmed Baba ${ }^{2}$, \\ Showkat Maqbool ${ }^{3}$, Mohammad Yousuf Zargar ${ }^{4}$ and Sajad Un Nabi ${ }^{5^{*}}$
}

\author{
${ }^{1}$ Division of Soil Science and Agricultural Chemistry FOA, Wadura, SKUAST -K, India \\ ${ }^{2}$ Biofertilizer Research Laboratory, FOA, Wadura, SKUAST -K, India \\ ${ }^{3}$ Division of Agri-economics and Statistics, FOA, Wadura, SKUAST-K, India \\ ${ }^{4}$ Directorate of research, SKUAST -K, India \\ ${ }^{5}$ ICAR-CITH, Old Airfield Rangreth, Srinagar, India
}

*Corresponding author

\section{A B S T R A C T}

Land use is characterized by the arrangements, activities and inputs that people undertake in a certain land cover type to produce change. It affects soil properties, overall soil health and the distribution supply of soil nutrients by directly altering biological transformations in the rooting zone. The present study "Assessment of soil microbial status under different

\section{Keywords}

Land use system, Microbial count, Bacteria, Kashmir, Soil

\section{Article Info}

Accepted:

04 July 2018

Available Online:

10 August 2018 land use systems in North western zone of Kashmir" was carried out to ascertain the biological properties of soils under five land use systems, viz., Forestry, Horticulture, Agriculture, Agri-Horti, Pasture at different locations viz., Gulmarg, Pattan, Ruhama, Baramulla, Sopore. The results revealed that, contrary to agro-ecosystem soils, forest showed the highest bacterial counts (cfu $\times 10^{6} / \mathrm{g}$ soil) with mean value of (178.46) while, the lowest (68.60) was found in agriculture land use system. The highest fungal count (cfu $\times 10^{5} / \mathrm{g}$ soil) with mean value (96.53) was observed in forest land use system and lowest $(16.20)$ in agriculture land use. The highest actinomycetes count ( $\mathrm{cfu} \times 10^{5} / \mathrm{g}$ soil) with mean value (73.86) was found in forest land use and lowest count (9.66) in agriculture land use. The highest azotobacter count ( $\mathrm{cfu} \times 10^{5} / \mathrm{g}$ soil) was found in forest land use with mean value of (63.53) and lowest in agriculture (8.33). The highest phosphate solubilizing bacteria count ( $\mathrm{cfu} \times 10^{5} / \mathrm{g}$ soil) was found in forest land use with mean value of (33.06) and lowest in agriculture (7.53). Our study confirmed that, the highest microbial count was found in forest soils and lowest in agriculture soils, probably because of presence of larger carbon source in the form of organic matter present in the forest soils as compared to other land use systems.

\section{Introduction}

Land use is characterized by the arrangements, activities and inputs, that people undertake in a certain land cover type to produce change or maintain it (Abad et al., 2014). It influences soil aggregation, aggregate stability and overall soil health and practices affect the distribution and supply of soil nutrients by directly altering soil properties and there by 
influencing biological transformations in the rooting zone (Nisar and Lone, 2013). The conversion of Forest Reserve to other land uses in recent times has caused many complex changes in the forest ecosystem whose impact raises diverse ecological problems (Awotoye et al., 2013). The conversion of forests into agriculture land had negative impacts on soil and diversely affects soil Carbon and Nitrogen levels (Michel et al., 2010).

Diverse microbial communities are supported by soil that plays an important role in ecosystem level processes such as, decomposition of organic matter and nutrient cycling. One cubic meter of soil may house many hundreds of species of bacteria, actinomycetes, fungi, and algae. The richness, abundance and activity of the microbial community is vulnerable to influence by soil physical and chemical properties such as $\mathrm{pH}$, moisture, organic matter content, and nutrient availability.

Alterations in the physical and chemical nature of the soil may lead to change in soil microbial community, composition, number and changes in microbial function (Bello et al., 2013). Land use systems such as forestry, pasture, cultivated land etc., provide stability and sustainability to the farming system.

The main aim of the study was to determine the effects of land use on the nature and population of microorganisms as soil microbial communities are susceptible to changes with different agricultural practices and land use management. Therefore, the present investigation entitled "Assessment of soil microbial status under different land use systems in North western zone of Kashmir" was carried out in district Baramulla of Jammu and Kashmir as the area has witnessed changes in the land use, for instance, the conversion of forests/pastures into cropland or a shift from agriculture to horticulture use.

\section{Materials and Methods}

\section{Location of the investigated area}

The study entitled "Assessment of soil biological properties under different land use systems in North western zone of Kashmir" has been carried out in district Baramulla of Jammu and Kashmir. The district is situated at $34.1980^{\circ} \mathrm{N}$ Longitude and $74.3636^{\circ} \mathrm{E}$ Latitude (Fig 1), at an average elevation of 1593 meters (5226 feet). The area encompasses many land uses and among them five dominant land uses are agriculture, forest, pasture, agri-horti and horticulture land use systems.

\section{Site selection and collection of soil samples}

Five land use systems viz., Forestry, Horticulture (Vegetables and Fruits), Agriculture, Agri-Horti, Pasture as shown in Fig. 2 at five different locations viz., Gulmarg, Pattan, Ruhama, Baramulla, Sopore of district Baramulla were identified for present study. The description of sampling sites under different land use systems is given in Table 1 and Fig 3. From these locations three sites per land use system were identified for collection of soil sample. Purposive sampling method was followed for collection of composite soil samples from each site with three replicates from a depth of $0-20 \mathrm{~cm}$ (Maqbool et al., 2017). The field moist soil samples were stored in refrigerator at temperature less than $4^{\circ} \mathrm{C}$ for determining the biological properties using standard procedures (sofi et al., 2016)

\section{Estimation of total microbial count}

Serial dilution pour plate technique by (Aneja, 2001) was used for estimation of total bacterial, fungal, actinomycetes, azotobacter, phosphate solubilizing bacteria using specific media i.e., nutrient agar media (NA), Martins Rose Bengal media, Actinomycetes agar, 
Ashbys media, pikovaskayas media respectively. One gram of the rhizosphere soil was placed in $9 \mathrm{ml}$ of sterilized distilled water under aseptic conditions. Serial dilution of $10^{2}, 10^{3}, 10^{4}, 10^{5}, 10^{6}$ were prepared. One $\mathrm{ml}$ of aliquot from specific dilution was added over cooled and solidified nutrient media (NA) in petriplates. The plates were rotated for uniform distribution. The plates were incubated at temperatures specific to particular microbe for 2-3 days. The colonies that developed on media were counted by electronic colony counter. Three replications were taken for each sample. The bacterial count was expressed as colony forming unit per gram of soil (Cfu/g soil).

\section{Statistical analysis}

Statistical analysis was carried out by using standard statistical procedures of Gomez and Gomez (1984) and SPSS window version 20.0 (SPSS Inc., Chicago, USA) packages.

\section{Results and Discussion}

\section{Total Bacterial Count}

The data (Table 2) revealed, that the total viable bacterial count ranged from 8.00 to $244.00 \mathrm{cfu} \times 10^{6} / \mathrm{g}$ soil. Contrary to agroecosystem soils, forest and pasture showed the highest bacterial counts (Fig 4). The highest bacterial count $\left(\mathrm{cfu} \times 10^{6} / \mathrm{g}\right.$ soil) was found in forest land use with mean value of (178.46) followed by pasture (173.86), horticulture (vegetables) (168.46), agri-horti (158.53), horticulture (fruits) (117.86).While, the lowest (68.60) was found in agriculture land use.

The number of soil bacteria in the cultivated land was lower than that in the other land use systems. This is probably because of presence of larger carbon source in the form of organic matter present in the forest and pasture land. This carbon source needed for metabolism may have increased the growth and activities of bacteria in soils of these land use systems. The slightly lower $\mathrm{pH}$ of the uncultivated land use type may have also encouraged the growth of bacteria which strive in that level of soil $\mathrm{pH}$. Soil $\mathrm{pH}$ has been shown to greatly influence soil microbial community (Ibekwe et al., 2012). Similar results that bacterial count was significantly affected by different land use system and conditions, and the highest bacterial count was found in surface soils of forest land use, grassland and lowest in cultivated land was also reported by Bello et al., (2013), Asadu et al., (2015), Kumar et al., (2017)

\section{Total fungal count}

The mean value of total viable fungi count (cfu $\times 10^{5} / \mathrm{g}$ soil) is presented in (Table 2$)$. The lowest (16.20) was recorded in agriculture land use and highest (96.53) was related in forest followed by pasture (63.46), horticulture (vegetables) (52.33), agri-horti (44.53), horticulture (fruits) (32.40). The mean fungal count forest soils were generally much higher than the other soils in the study area (Fig 5). The total viable fungal count ranged from 0.00 to $120.00 \mathrm{cfu} \times 10^{5} / \mathrm{g}$ soil. It might be due to low $\mathrm{pH}$ and higher organic matter in the forest soils. It is also possible that the presence of trees in this land use system may have encouraged the presence of ectomycorrhizal fungi which colonize most tree species whereas changes in soil physical properties resulting from tillage operations common in cultivated land may have equally contributed to the reduced number of fungi in the cultivated land. This is because fungi are easily influenced by changes in soil and environmental conditions (Sui et al., 2012). Fungal structure (hyphal growth) is greatly affected by conventional agricultural practices. Furthermore, the presence of trees in the forestland may have reduced the impact of heavy rainfall and other climatic variables thus, favoring abundant growth of fungi in the forest land Asadu et al., (2015). 
Table.1 Description of sampling sites under different Land Use Systems of District Baramulla

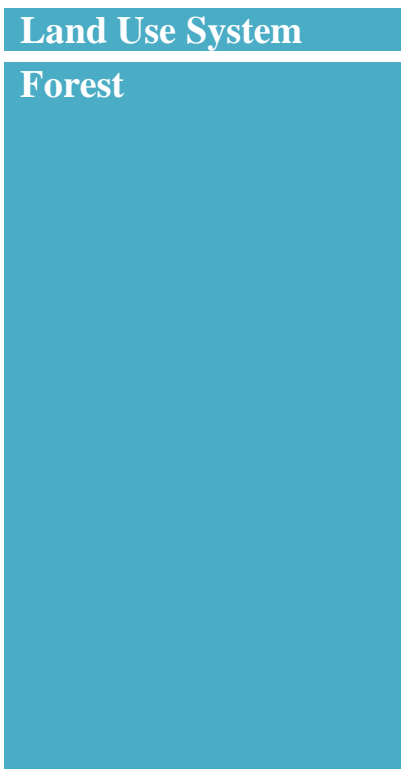

\section{Horticulture}

Vegetable

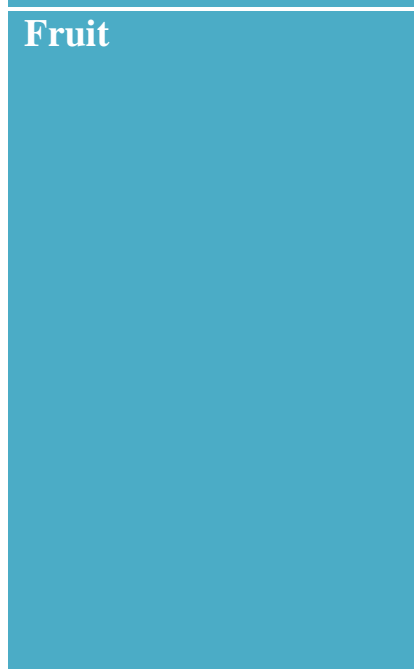

\begin{tabular}{|c|c|c|}
\hline Location & Site & Site No. \\
\hline \multirow[t]{3}{*}{ Tangmarg } & $\mathrm{C}-37$ & S1 \\
\hline & C-39 & S2 \\
\hline & Chamdinala & S3 \\
\hline \multirow[t]{3}{*}{ Pattan } & Palhalan & S1 \\
\hline & Reengh & S2 \\
\hline & Parihaspora & S3 \\
\hline \multirow[t]{3}{*}{ Ruhama } & Rawchha & S1 \\
\hline & Sangrampora & S2 \\
\hline & Mandinu & S3 \\
\hline \multirow[t]{3}{*}{ Baramulla main } & Veeriwan & $\mathrm{S} 1$ \\
\hline & Sheeri & S2 \\
\hline & Sheeri & S3 \\
\hline \multirow[t]{3}{*}{ Sopore } & Zaloora & S1 \\
\hline & Harwan & S2 \\
\hline & Latishat & S3 \\
\hline \multirow[t]{3}{*}{ Tangmarg } & Gokhama & S1 \\
\hline & Kunzer & S2 \\
\hline & Tangmarg & S3 \\
\hline \multirow[t]{3}{*}{ Pattan } & Palhalan & S1 \\
\hline & Hanjura & $\mathrm{S} 2$ \\
\hline & Zangam & S3 \\
\hline \multirow[t]{3}{*}{ Ruhama } & Tragpora & $\mathrm{S} 1$ \\
\hline & Dangiwacha & S2 \\
\hline & Ladura & S3 \\
\hline \multirow[t]{3}{*}{ Baramulla main } & Delina & S1 \\
\hline & Arampora & $\mathrm{S} 2$ \\
\hline & Johama & S3 \\
\hline \multirow[t]{3}{*}{ Sopore } & Samad-Abad & $\mathrm{S} 1$ \\
\hline & Jalalabad & $\mathrm{S} 2$ \\
\hline & Mazbug & S3 \\
\hline \multirow[t]{3}{*}{ Tangmarg } & Gokhama & $\mathrm{S} 1$ \\
\hline & Kunzer & $\mathrm{S} 2$ \\
\hline & Tangmarg & S3 \\
\hline \multirow[t]{3}{*}{ Pattan } & Gooin & $\mathrm{S} 1$ \\
\hline & Hanjura & S2 \\
\hline & Zangam & S3 \\
\hline \multirow[t]{3}{*}{ Ruhama } & Sangrampora & S1 \\
\hline & Dangiwacha & $\mathrm{S} 2$ \\
\hline & Hadipora & S3 \\
\hline \multirow[t]{3}{*}{ Baramulla main } & Veeriwan & S1 \\
\hline & Johama & $\mathrm{S} 2$ \\
\hline & Nadihal & S3 \\
\hline \multirow[t]{2}{*}{ Sopore } & Saidpora & $\mathrm{S} 1$ \\
\hline & Shangergund & $\mathrm{S} 2$ \\
\hline
\end{tabular}



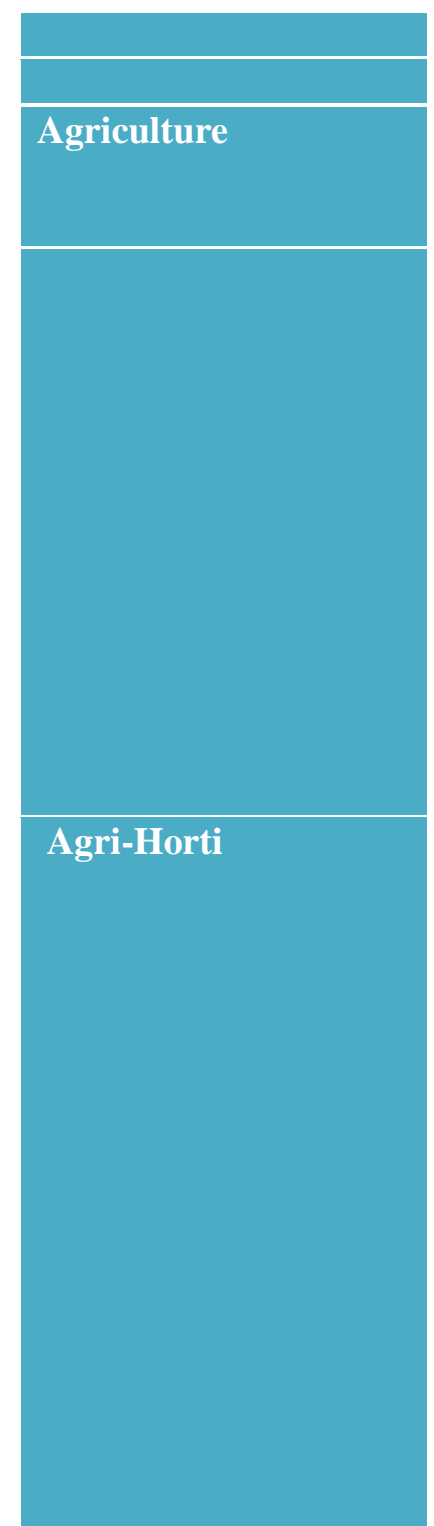

Pasture

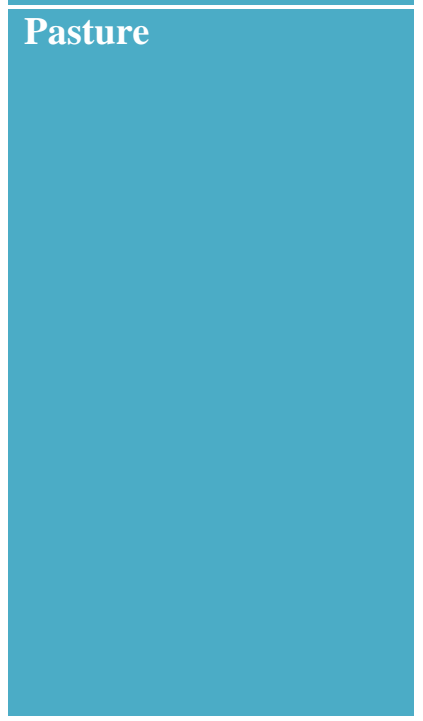

\begin{tabular}{|c|c|c|}
\hline & Hygam & S3 \\
\hline \multirow{3}{*}{ Tangmarg } & Druroo & S1 \\
\hline & Shraie & S2 \\
\hline & Reram & S3 \\
\hline \multirow[t]{3}{*}{ Pattan } & Gooin & S1 \\
\hline & Hanjura & S2 \\
\hline & Zangam & S3 \\
\hline \multirow[t]{3}{*}{ Ruhama } & Hachivi Pora & S1 \\
\hline & Batsum & S2 \\
\hline & Watrigam & S3 \\
\hline \multirow[t]{3}{*}{ Baramulla main } & Johama & S1 \\
\hline & Sheeri & S2 \\
\hline & Chakla & S3 \\
\hline \multirow[t]{3}{*}{ Sopore } & Samad-Abad & S1 \\
\hline & Shiva & S2 \\
\hline & Daranambal & S3 \\
\hline \multirow[t]{3}{*}{ Tangmarg } & Shraie & $\mathrm{S} 1$ \\
\hline & Reram & $\mathrm{S} 2$ \\
\hline & Sadkipora reram & S3 \\
\hline \multirow[t]{3}{*}{ Pattan } & Paripora & S1 \\
\hline & Palhalan & S2 \\
\hline & Palhalan & S3 \\
\hline \multirow[t]{3}{*}{ Ruhama } & Hadipora & S1 \\
\hline & Ladura & $\mathrm{S} 2$ \\
\hline & Tragpora & S3 \\
\hline \multirow[t]{3}{*}{ Baramulla main } & Arampora & $\mathrm{S} 1$ \\
\hline & Johama & S2 \\
\hline & Bear & S3 \\
\hline \multirow[t]{3}{*}{ Sopore } & Brath & $\mathrm{S} 1$ \\
\hline & Goripora & S2 \\
\hline & Botingoo & S3 \\
\hline \multirow[t]{3}{*}{ Tangmarg } & Gulmarg pasture-1 & S1 \\
\hline & Gulmarg pasture-2 & S2 \\
\hline & Gulmarg pasture-3 & S3 \\
\hline \multirow[t]{3}{*}{ Pattan } & Hyderbeigh & S1 \\
\hline & Palhalan ghat & $\mathrm{S} 2$ \\
\hline & Tapeer payeen & S3 \\
\hline \multirow[t]{3}{*}{ Ruhama } & Behrampora & S1 \\
\hline & Panzala & S2 \\
\hline & Khusipora & S3 \\
\hline \multirow[t]{3}{*}{ Baramulla main } & Darpora Delina & S1 \\
\hline & Delina & S2 \\
\hline & Janbazpora & S3 \\
\hline \multirow[t]{3}{*}{ Sopore } & Warpora & S1 \\
\hline & Nengli & S2 \\
\hline & Hanjipora Hygam & S3 \\
\hline
\end{tabular}


Table.2 Microbial count of soil under different Land Use Systems

\begin{tabular}{|c|c|c|c|c|c|c|c|}
\hline LUS & Location & Site & $\begin{array}{l}\text { Total viable } \\
\text { Bacteria } \\
\text { cfu } \times 10^{6} \mathrm{~g}^{-1} \text { soil }\end{array}$ & $\begin{array}{l}\text { Total viable Fungi } \\
\text { cfux } 10^{5} \mathrm{~g}^{1} \text { soil }\end{array}$ & $\begin{array}{l}\text { Total viable } \\
\text { Actinomycetes } \\
\text { cfu } \times 10^{5} \mathrm{~g}^{-1} \text { soil }\end{array}$ & $\begin{array}{l}\text { Azotobacter } \\
\text { cfu } \times 10^{5} \mathrm{~g}^{-1} \text { soil }\end{array}$ & $\begin{array}{l}\text { Phosphate } \\
\text { solubilizing bacteria } \\
\text { cfu } \times 10^{5} \mathrm{~g}^{-1} \text { soil }\end{array}$ \\
\hline \multirow[t]{15}{*}{ Forestry } & Tangmarg & $\mathrm{S}_{1}$ & 240 & 120 & 88 & 72 & 32 \\
\hline & & $\mathrm{S}_{2}$ & 236 & 108 & 76 & 64 & 36 \\
\hline & & $\mathrm{S}_{3}$ & 244 & 116 & 84 & 68 & 20 \\
\hline & Pattan & $S_{1}$ & 232 & 88 & 72 & 60 & 16 \\
\hline & & $\mathrm{S}_{2}$ & 116 & 92 & 80 & 76 & 24 \\
\hline & & $\mathrm{S}_{3}$ & 228 & 103 & 86 & 80 & 76 \\
\hline & Ruhama & $\mathrm{S}_{1}$ & 108 & 100 & 92 & 84 & 8 \\
\hline & & $\mathrm{S}_{2}$ & 104 & 112 & 96 & 81 & 28 \\
\hline & & $\mathrm{S}_{3}$ & 221 & 96 & 68 & 56 & 12 \\
\hline & Baramulla & $\mathrm{S}_{1}$ & 112 & 84 & 52 & 48 & 44 \\
\hline & & $\mathrm{S}_{2}$ & 119 & 76 & 64 & 52 & 40 \\
\hline & & $\mathrm{S}_{3}$ & 159 & 80 & 56 & 40 & 32 \\
\hline & Sopore & $\mathrm{S}_{1}$ & 231 & 97 & 60 & 36 & 28 \\
\hline & & $\mathrm{S}_{2}$ & 92 & 104 & 77 & 76 & 52 \\
\hline & & $\mathrm{S}_{3}$ & 235 & 72 & 57 & 60 & 48 \\
\hline $\begin{array}{l}\text { Range } \\
\text { Mean } \pm \text { S.E } \\
95 \% \text { C.I } \\
\end{array}$ & & & $\begin{array}{l}92.00-244.00 \\
178.46 \pm 16.13 \\
143.85-213.08\end{array}$ & $\begin{array}{l}\text { 72.00-120.00 } \\
96.53 \pm 3.75 \\
88.48-104.58\end{array}$ & $\begin{array}{l}52.00-96.00 \\
73.86 \pm 3.60 \\
66.13-81.59\end{array}$ & $\begin{array}{l}\text { 36.00-84.00 } \\
63.53 \pm 3.88 \\
55.20-71.86\end{array}$ & $\begin{array}{l}8.00-76.00 \\
33.06 \pm 4.50 \\
23.39-42.73\end{array}$ \\
\hline Horticulture & Tangmarg & $\mathrm{S}_{1}$ & 201 & 61 & 45 & 39 & 37 \\
\hline \multirow[t]{14}{*}{ 1)Vegetables } & & $\mathrm{S}_{2}$ & 159 & 69 & 64 & 55 & 53 \\
\hline & & $\mathrm{S}_{3}$ & 205 & 33 & 29 & 23 & 21 \\
\hline & Pattan & $S_{1}$ & 150 & 37 & 25 & 19 & 17 \\
\hline & & $\mathrm{S}_{2}$ & 141 & 49 & 37 & 31 & 25 \\
\hline & & $\mathrm{S}_{3}$ & 189 & 41 & 21 & 15 & 9 \\
\hline & Ruhama & $\mathrm{S}_{1}$ & 137 & 57 & 13 & 7 & 2 \\
\hline & & $\mathrm{S}_{2}$ & 143 & 71 & 41 & 35 & 33 \\
\hline & & $\mathrm{S}_{3}$ & 133 & 68 & 49 & 43 & 37 \\
\hline & Baramulla & $\mathrm{S}_{1}$ & 145 & 86 & 53 & 39 & 29 \\
\hline & & $\mathrm{S}_{2}$ & 173 & 65 & 57 & 47 & 45 \\
\hline & & $\mathrm{S}_{3}$ & 221 & 53 & 33 & 10 & 13 \\
\hline & Sopore & $S_{1}$ & 210 & 45 & 44 & 29 & 17 \\
\hline & & $\mathrm{S}_{2}$ & 191 & 29 & 25 & 0 & 9 \\
\hline & & $\mathrm{S}_{3}$ & 129 & 21 & 17 & 7 & 4 \\
\hline
\end{tabular}




\begin{tabular}{|c|c|c|c|c|c|c|c|}
\hline $\begin{array}{l}\text { Range } \\
\text { Mean } \pm \text { S.E } \\
95 \% \text { C.I } \\
\end{array}$ & & & $\begin{array}{l}129.00-221.00 \\
168.46 \pm 8.17 \\
150.94-186.00\end{array}$ & $\begin{array}{l}21.00-86.00 \\
52.33 \pm 4.67 \\
42.30-62.36\end{array}$ & $\begin{array}{l}13.00-64.00 \\
36.86 \pm 3.93 \\
28.41-45.31\end{array}$ & $\begin{array}{l}0.00-55.00 \\
26.60 \pm 4.28 \\
17.41-35.78\end{array}$ & $\begin{array}{l}2.00-53.00 \\
23.40 \pm 3.95 \\
14.91-31.88\end{array}$ \\
\hline \multirow[t]{15}{*}{ 2)Fruits } & Tangmarg & $\mathrm{S}_{1}$ & 100 & 44 & 30 & 20 & 12 \\
\hline & & $\mathrm{S}_{2}$ & 104 & 16 & 13 & 8 & 4 \\
\hline & & $\mathrm{S}_{3}$ & 96 & 52 & 40 & 36 & 32 \\
\hline & Pattan & $\mathrm{S}_{1}$ & 108 & 28 & 19 & 12 & 8 \\
\hline & & $\mathrm{S}_{2}$ & 124 & 32 & 10 & 21 & 0 \\
\hline & & $\mathrm{S}_{3}$ & 128 & 48 & 37 & 28 & 20 \\
\hline & Ruhama & $\mathrm{S}_{1}$ & 107 & 20 & 14 & 3 & 0 \\
\hline & & $\mathrm{S}_{2}$ & 201 & 18 & 16 & 10 & 3 \\
\hline & & $\mathrm{S}_{3}$ & 127 & 24 & 0 & 4 & 9 \\
\hline & Baramulla & $\mathrm{S}_{1}$ & 91 & 36 & 4 & 15 & 16 \\
\hline & & $\mathrm{S}_{2}$ & 101 & 43 & 20 & 16 & 11 \\
\hline & & $\mathrm{S}_{3}$ & 109 & 15 & 8 & 9 & 2 \\
\hline & Sopore & $\mathrm{S}_{1}$ & 99 & 30 & 12 & 0 & 13 \\
\hline & & $\mathrm{S}_{2}$ & 121 & 41 & 28 & 24 & 20 \\
\hline & & $\mathrm{S}_{3}$ & 152 & 39 & 32 & 30 & 27 \\
\hline $\begin{array}{l}\text { Range } \\
\text { Mean } \pm \text { S.E } \\
95 \% \text { C.I } \\
\end{array}$ & & & $\begin{array}{l}91.00-201.00 \\
117.86 \pm 7.22 \\
102.37-133.36\end{array}$ & $\begin{array}{l}15.00-52.00 \\
32.40 \pm 3.11 \\
25.72-39.07\end{array}$ & $\begin{array}{l}0.00-40.00 \\
18.86 \pm 3.11 \\
12.17-25.55\end{array}$ & $\begin{array}{l}0.00-36.00 \\
15.73 \pm 2.74 \\
9.85-21.61\end{array}$ & $\begin{array}{l}0.00-32.00 \\
11.80 \pm 2.51 \\
6.40-17.19\end{array}$ \\
\hline \multirow[t]{15}{*}{ Agriculture } & Tangmarg & $\mathrm{S}_{1}$ & 96 & 16 & 8 & 4 & 4 \\
\hline & & $\mathrm{S}_{2}$ & 92 & 4 & 4 & 8 & 2 \\
\hline & & $\mathrm{S}_{3}$ & 73 & 20 & 12 & 0 & 8 \\
\hline & Pattan & $\mathrm{S}_{1}$ & 84 & 8 & 3 & 1 & 12 \\
\hline & & $\mathrm{S}_{2}$ & 91 & 19 & 15 & 9 & 3 \\
\hline & & $\mathrm{S}_{3}$ & 76 & 28 & 20 & 12 & 7 \\
\hline & Ruhama & $\mathrm{S}_{1}$ & 88 & 12 & 0 & 3 & 20 \\
\hline & & $\mathrm{S}_{2}$ & 62 & 20 & 17 & 11 & 16 \\
\hline & & $\mathrm{S}_{3}$ & 89 & 31 & 0 & 5 & 2 \\
\hline & Baramulla & $\mathrm{S}_{1}$ & 58 & 43 & 30 & 16 & 14 \\
\hline & & $\mathrm{S}_{2}$ & 72 & 24 & 13 & 7 & 0 \\
\hline & & $\mathrm{S}_{3}$ & 80 & 0 & 7 & 13 & 9 \\
\hline & Sopore & $\mathrm{S}_{1}$ & 8 & 3 & 0 & 17 & 10 \\
\hline & & $\mathrm{S}_{2}$ & 20 & 15 & 11 & 0 & 6 \\
\hline & & $\mathrm{S}_{3}$ & 40 & 0 & 5 & 19 & 0 \\
\hline $\begin{array}{l}\text { Range } \\
\text { Mean } \pm \text { S.E }\end{array}$ & & & $\begin{array}{l}8.00-96.00 \\
68.60 \pm 6.91\end{array}$ & $\begin{array}{l}0.00-43.00 \\
16.20 \pm 3.16\end{array}$ & $\begin{array}{l}0.00-30.00 \\
9.66 \pm 2.19\end{array}$ & $\begin{array}{l}0.00-19.00 \\
8.33 \pm 1.60\end{array}$ & $\begin{array}{l}0.00-20.00 \\
7.53 \pm 1.55\end{array}$ \\
\hline
\end{tabular}




\begin{tabular}{|c|c|c|c|c|c|c|c|}
\hline 95\% C.I & & & $53.76-83.44$ & $9.40-22.99$ & 4.96-14.36 & 4.88-11.78 & $4.19-10.86$ \\
\hline \multirow[t]{15}{*}{ Agri-Horti } & Tangmarg & $\mathrm{S}_{1}$ & 200 & 57 & 40 & 36 & 37 \\
\hline & & $\mathrm{S}_{2}$ & 156 & 64 & 56 & 52 & 48 \\
\hline & & $\mathrm{S}_{3}$ & 204 & 28 & 24 & 20 & 16 \\
\hline & Pattan & $\mathrm{S}_{1}$ & 135 & 32 & 20 & 16 & 11 \\
\hline & & $\mathrm{S}_{2}$ & 148 & 44 & 32 & 28 & 20 \\
\hline & & $\mathrm{S}_{3}$ & 150 & 36 & 17 & 12 & 4 \\
\hline & Ruhama & $\mathrm{S}_{1}$ & 132 & 52 & 8 & 20 & 0 \\
\hline & & $\mathrm{S}_{2}$ & 137 & 43 & 36 & 32 & 28 \\
\hline & & $\mathrm{S}_{3}$ & 128 & 68 & 44 & 40 & 32 \\
\hline & Baramulla & $\mathrm{S}_{1}$ & 140 & 56 & 48 & 0 & 24 \\
\hline & & $\mathrm{S}_{2}$ & 152 & 60 & 52 & 44 & 40 \\
\hline & & $\mathrm{S}_{3}$ & 216 & 48 & 28 & 17 & 8 \\
\hline & Sopore & $\mathrm{S}_{1}$ & 208 & 40 & 36 & 8 & 12 \\
\hline & & $\mathrm{S}_{2}$ & 148 & 24 & 19 & 21 & 21 \\
\hline & & $\mathrm{S}_{3}$ & 124 & 16 & 12 & 4 & 0 \\
\hline $\begin{array}{l}\text { Range } \\
\text { Mean } \pm \text { S.E } \\
95 \% \text { C.I } \\
\end{array}$ & & & $\begin{array}{l}124.00-216.00 \\
158.53 \pm 8.10 \\
140.97-176.08\end{array}$ & $\begin{array}{l}16.00-68.00 \\
44.53 \pm 3.95 \\
36.04-53.01\end{array}$ & $\begin{array}{l}8.00-56.00 \\
31.46 \pm 3.81 \\
23.27-39.65\end{array}$ & $\begin{array}{l}0.00-52.00 \\
23.33 \pm 3.89 \\
14.97-31.68\end{array}$ & $\begin{array}{l}0.00-48.00 \\
20.06 \pm 3.81 \\
11.88-28.25\end{array}$ \\
\hline \multirow[t]{15}{*}{ Pasture } & Tangmarg & $\mathrm{S}_{1}$ & 200 & 84 & 68 & 48 & 44 \\
\hline & & $\mathrm{S}_{2}$ & 232 & 68 & 0 & 29 & 32 \\
\hline & & $\mathrm{S}_{3}$ & 212 & 72 & 63 & 56 & 51 \\
\hline & Pattan & $\mathrm{S}_{1}$ & 157 & 60 & 50 & 30 & 48 \\
\hline & & $\mathrm{S}_{2}$ & 124 & 88 & 44 & 64 & 60 \\
\hline & & $\mathrm{S}_{3}$ & 141 & 60 & 52 & 52 & 42 \\
\hline & Ruhama & $\mathrm{S}_{1}$ & 212 & 56 & 76 & 32 & 27 \\
\hline & & $\mathrm{S}_{2}$ & 96 & 76 & 35 & 28 & 8 \\
\hline & & $\mathrm{S}_{3}$ & 216 & 84 & 51 & 12 & 16 \\
\hline & Baramulla & $\mathrm{S}_{1}$ & 152 & 72 & 28 & 24 & 20 \\
\hline & & $\mathrm{S}_{2}$ & 215 & 96 & 32 & 0 & 11 \\
\hline & & $\mathrm{S}_{3}$ & 88 & 0 & 55 & 36 & 37 \\
\hline & Sopore & $\mathrm{S}_{1}$ & 236 & 48 & 41 & 0 & 3 \\
\hline & & $\mathrm{S}_{2}$ & 128 & 36 & 24 & 19 & 21 \\
\hline & & $\mathrm{S}_{3}$ & 199 & 52 & 30 & 16 & 52 \\
\hline $\begin{array}{l}\text { Range } \\
\text { Mean } \pm \text { S.E } \\
95 \% \text { C.I }\end{array}$ & & & $\begin{array}{c}88.00-236.00 \\
173.86 \pm 12.87 \\
146.25-201.48\end{array}$ & $\begin{array}{l}0.00-96.00 \\
63.46 \pm 6.19 \\
50.17-76.75\end{array}$ & $\begin{array}{l}0.00-76.00 \\
43.26 \pm 4.99 \\
32.54-53.98\end{array}$ & $\begin{array}{l}0.00-64.00 \\
29.73 \pm 4.95 \\
19.10-40.36\end{array}$ & $\begin{array}{l}3.00-60.00 \\
31.46 \pm 4.61 \\
21.57-41.36\end{array}$ \\
\hline
\end{tabular}


Fig.1 Map of study area

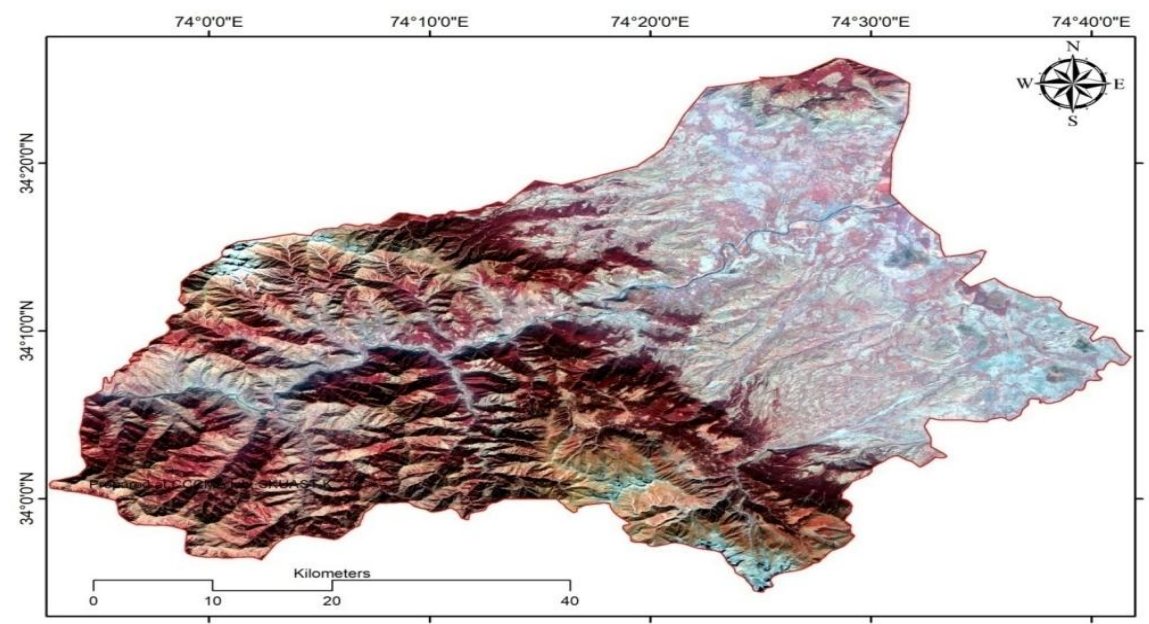

Fig.2 Map showing sampling sites under

Fig.3 Sampling sites Different land use systems
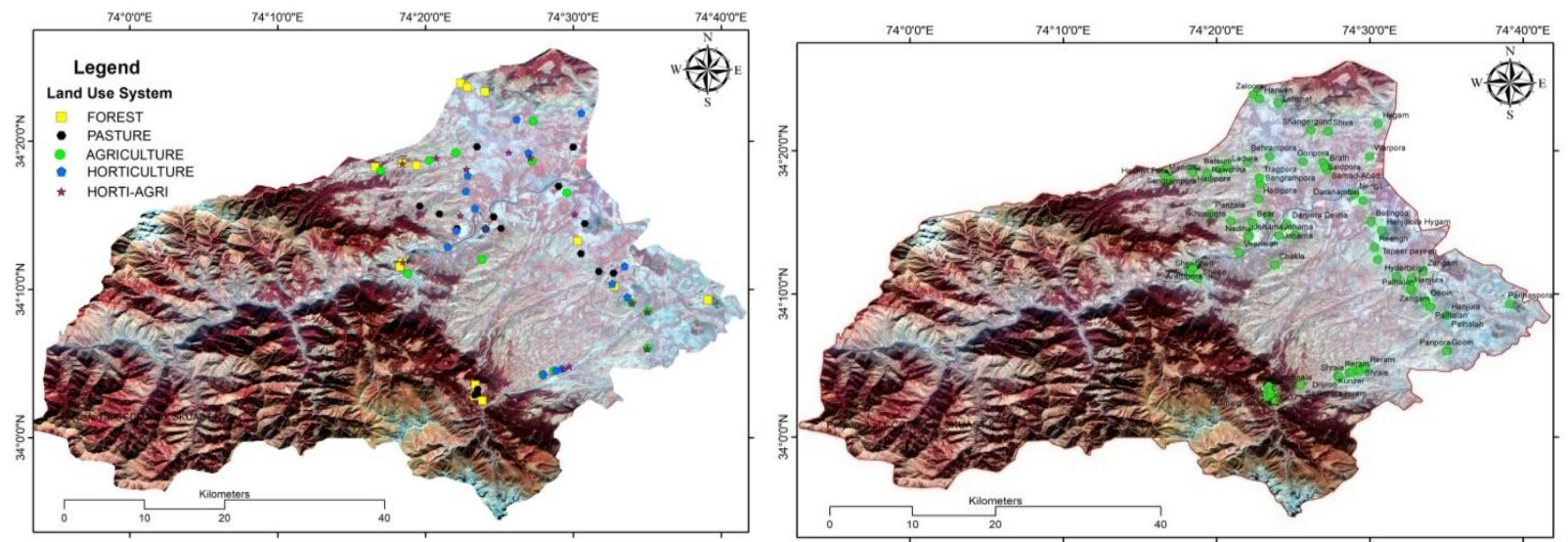

Fig.4 Graph showing bacterial count under different land uses systems

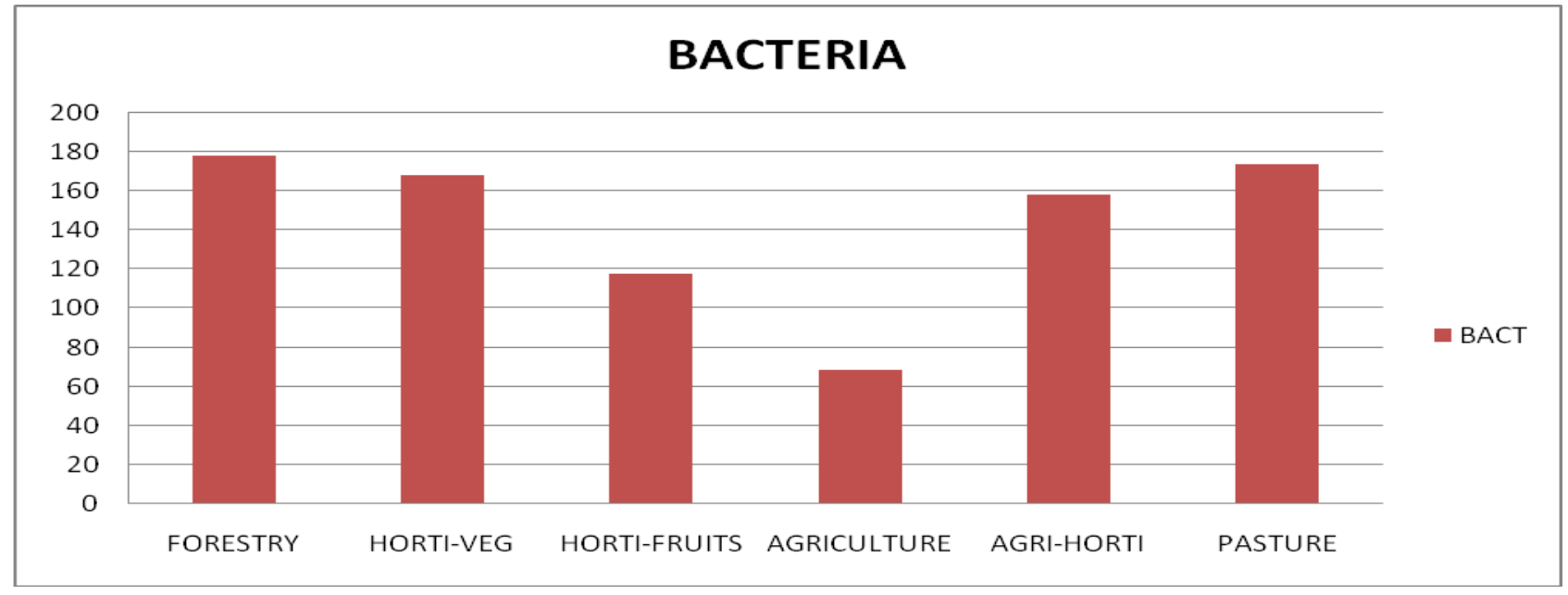


Fig.5 Graph showing fungal count under different land use systems

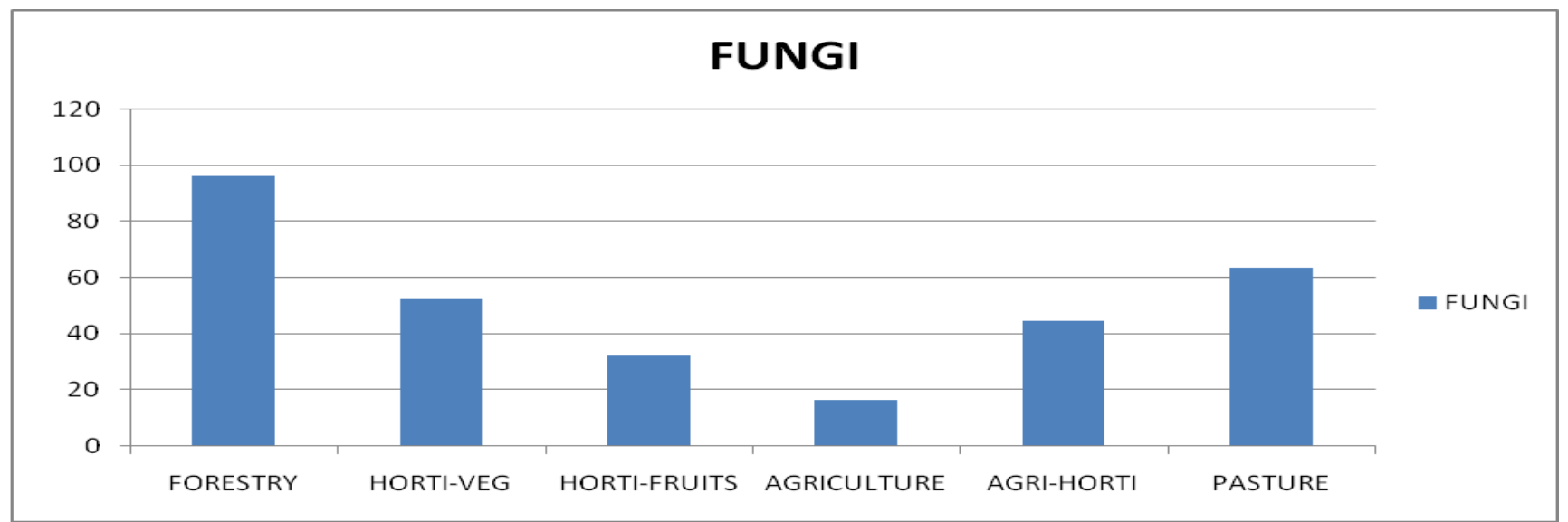

Fig.6 Graph showing Actinomycetes count under different land use systems

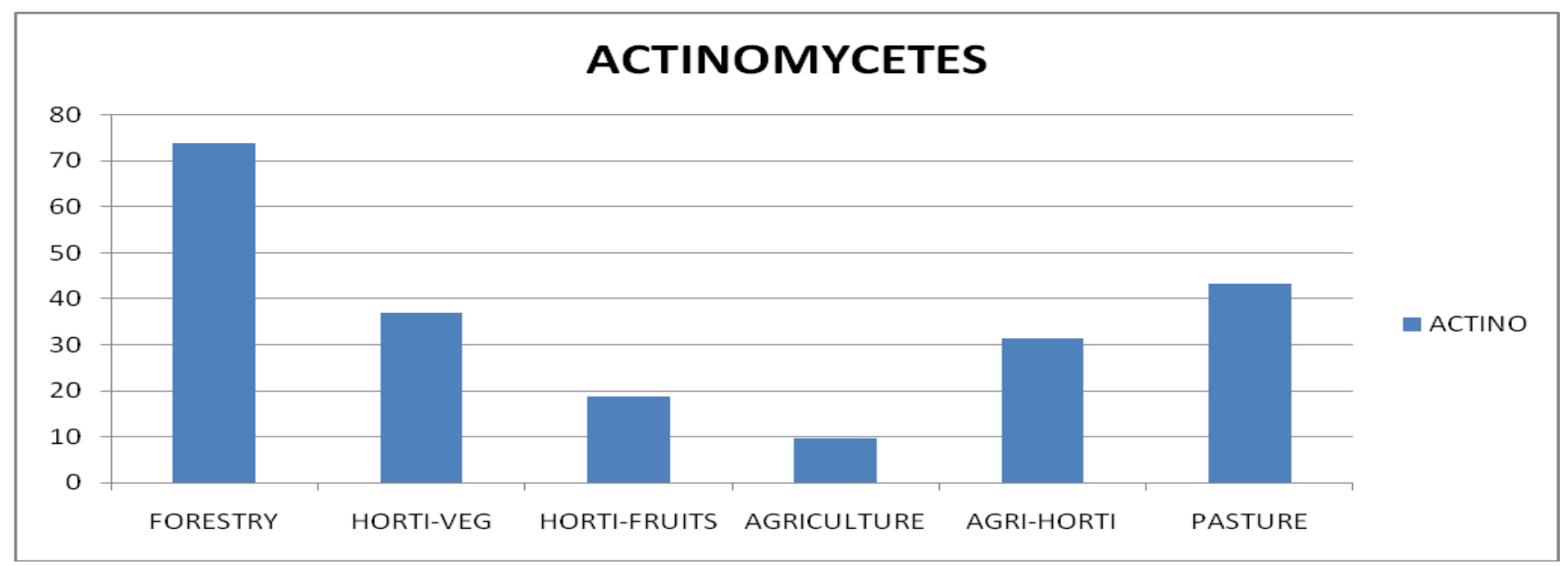

Fig.7 Graph showing Azotobacter count under different land use systems

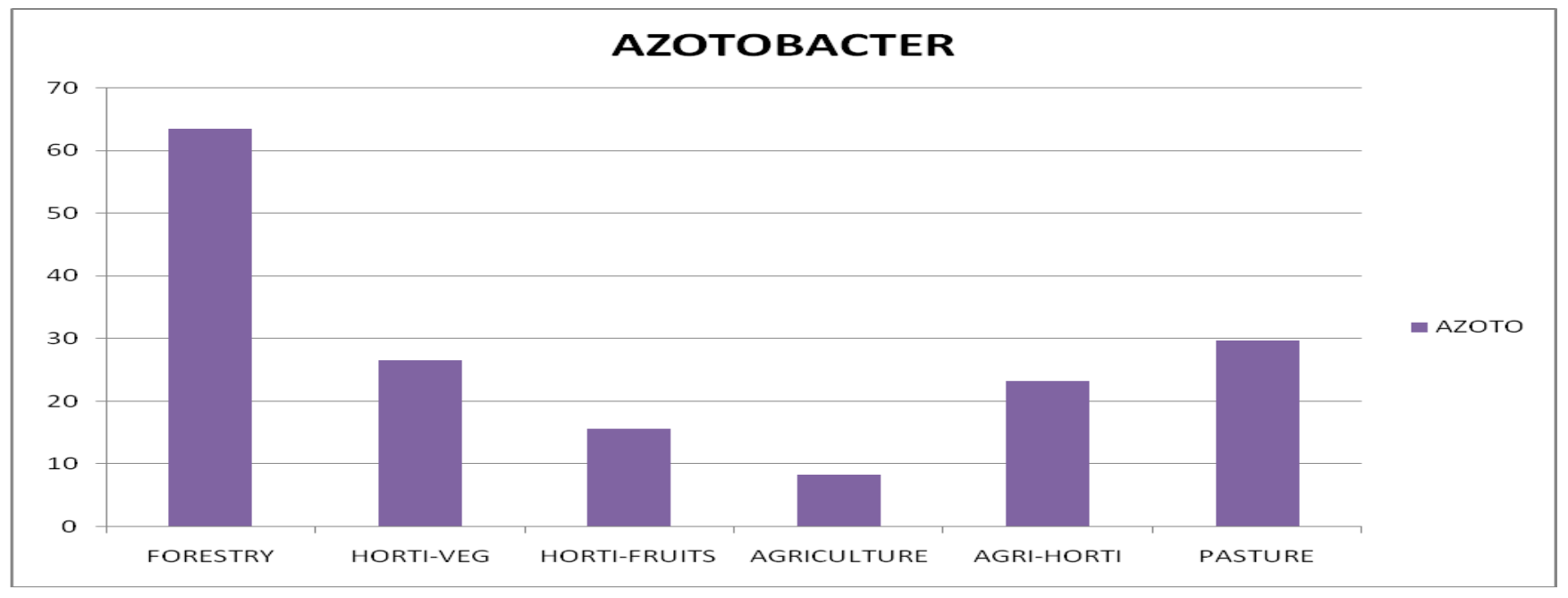


Fig.8 Graph showing PSB count under different land use systems

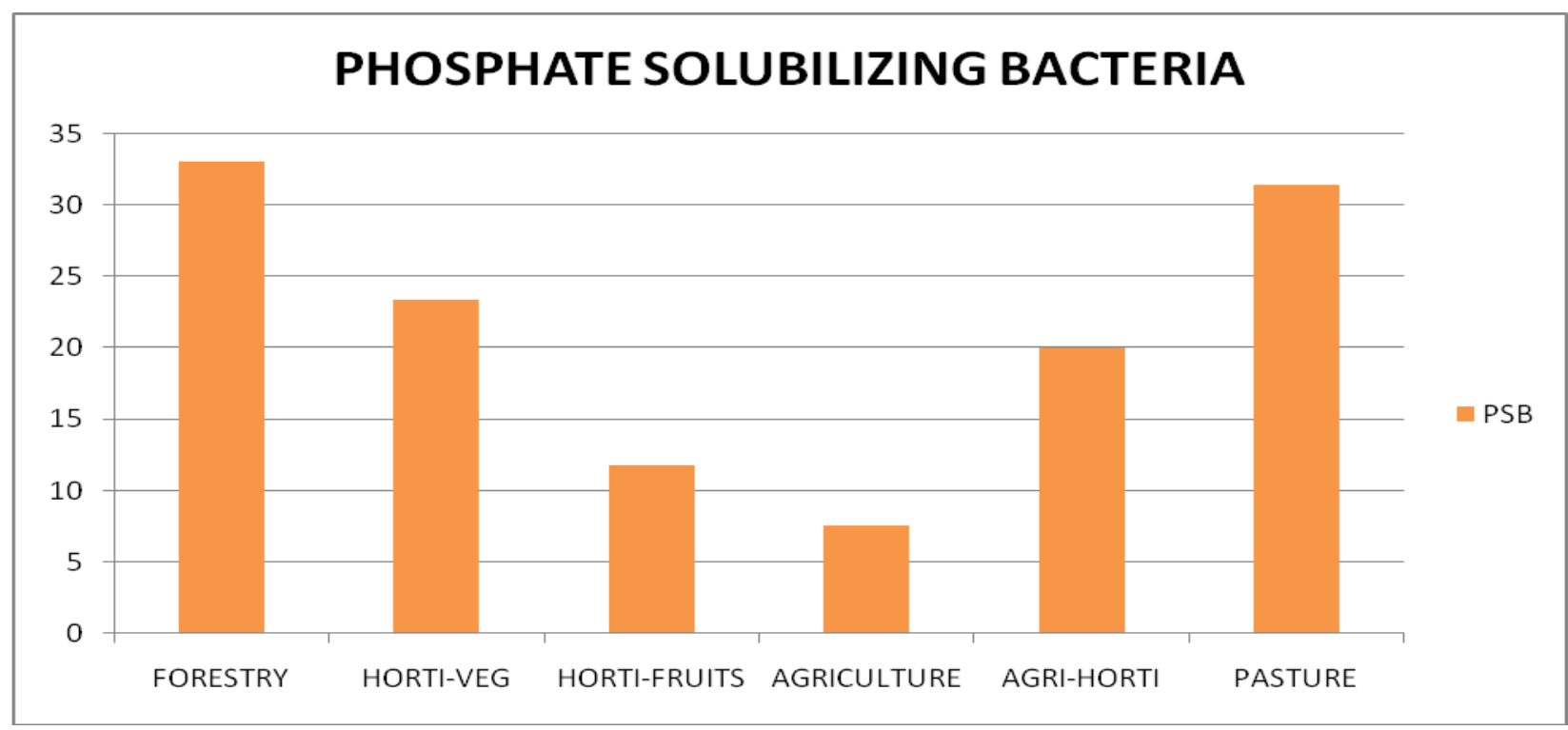

The results are in unison with the findings of Bello et al., (2013), Asadu et al., (2015), Kumar et al., (2017) who also reported that fungal count was significantly affected by different land use systems however, the highest fungal count of surface and subsurface soils was found in forest land.

\section{Total actinomycetes count}

The total viable actinomycetes count (cfu $\times 10^{5} / \mathrm{g}$ soil) ranged from $0.00-96.00$ (Table 2 ). The highest actinomycetes count $\left(\mathrm{cfu} \times 10^{5} / \mathrm{g}\right.$ soil) was found in forest land use (Fig 6) with mean value of (73.86), followed by pasture (43.26), horticulture (vegetables) (36.86), agri-horti (31.46). The lowest actinomycetes count (9.66) was found in agriculture land use as compared to other soils in the study area followed by horticulture (fruits) (18.86). This may be due to higher pore space as well as more organic material in forest and grassland which is added to the soil through leaf litter which serves as a source of energy for microbial population with good vegetation cover is reason for increased microbial activity. Moreover, the more activity of microorganisms in grassland and forests are also due to presence of more plant roots. The less microbial count in cultivated land is due to low organic matter and use of fertilizers and more tillage practices. The results corroborate the findings of Okonkwo (2010) who also reported that continuous cultivation led to a decrease in the population of bacteria, actinomycetes and algae. Kumar et al., (2017) also found that actinomycetes count was significantly affected by different land use system and conditions. However, the highest actinomycetes count of surface and subsurface soils was found in forest land use

\section{Total azotobacter count}

The perusal of data (Table 2) reveals that the total azotobacter count (cfu $\times 10^{5} / \mathrm{g}$ soil) ranged from (0.00-84.00). The highest count was observed in natural ecosystems as compared to cultivated lands in study area (Fig 7). The highest azotobacter count (cfu $\times 10^{5} / \mathrm{g}$ soil) was found in forest land use with mean value of (63.53) followed by pasture (29.73), horticulture (vegetables) (26.60) and lowest in agriculture (8.33), horticulture (fruits) (15.73), agri-horti (23.33). The highest microbial count in forests could be attributed 
to more organic matter in forest soils and its accumulation in soil which acts as food for microorganisms. Higher organic matter levels also result in favorable soil temperature; improve plant root growth and in turn healthy microbial populations. The results are in agreement with the findings of Bello et al., (2013), Asadu et al., (2015), Kumar et al., (2017). Maurya et al., (2012) also reported highest azotobacter population in agroforestry and lowest in cultivated lands.

\section{Total Phosphate solubilizing bacteria Count}

The phosphate solubilizing bacteria count (cfu $\times 10^{5} / \mathrm{g}$ soil) ranged from (0.00-76.00) in different land use systems (Table 2). The diversity in population density of phosphate solubilizing bacteria was maximum in soil of forestry followed by pasture and lowest in agriculture (Fig 8). The highest phosphate solubilizing bacteria count ( $\mathrm{cfu} \times 10^{5} / \mathrm{g}$ soil) was found in forest land use with mean value of (33.06) followed by pasture (31.46), horticulture (vegetables) (23.40), agri-horti (20.06), and lowest in agriculture (7.53), horticulture (fruits) (11.80). The low phosphate solubilizing bacteria population in cultivated land may be attributed to the reason that application of agrochemicals, especially pesticides might be associated with the population density declines of phosphate solubilizing bacteria as a response to changing of environmental conditions for microbial growth as soil microbes are very sensitive to external disturbances in their habitats. The application of pesticide can substantially affect populations and activity of phosphate solubilizing bacteria (Sethi et al., 2013) and biochemical processes including dissolution of soil phosphorus because of the negative impact of pesticide on the synthesis and metabolism of both enzymes and proteins (Srinivasulu and Rangaswamy, 2014). High population in forest soils may be due to higher pore space and organic material added to the soil through leaf litter which serves as a source of energy for microbial population.

Land use systems such as forestry, pasture, cultivated land etc., provide stability and sustainability to the farming system. It affects soil properties, overall soil health and the distribution supply of soil nutrients by directly altering biological transformations in the rooting zone. The present study under five land use systems, viz., Forestry, Horticulture, Agriculture, Agri-Horti, and Pasture at different locations revealed that the highest microbial count was found in forest soils and lowest in agriculture soils, probably because of presence of larger carbon source in the form of organic matter present in the forest soils as compared to other land use systems.

\section{References}

Abad, J.R., Khosravi, H. and Alamdarlou, E.H. 2014. Assessment the effects of land use changes on soil physicochemical properties in Jafarabad of Golestan province, Iran. Bulletin of Environment, Pharmacology and Life Sciences. 3 (3): 296-300.

Aneja, R.K. 2001. Experiments in Microbiology, Plant Pathology, tissue culture and mushroom production technology. Third edition. New age International Publishers, Pvt. New Delhi, pp. 568.

Asadu C. L. A., Nwafor I. A., Chibuike G. U. 2015. Contributions of Microorganisms to Soil Fertility in Adjacent Forest, Fallow and Cultivated Land Use Types in Nsukka, Nigeria. International Journal of Agriculture and Forestry. 5(3): 199-204

Awotoye. O.O, Adebola. S.I. and Matthew. O.J. 2013. The effects of land-use changes on soil properties in a humid tropical location; Little-Ose forest 
reserve, south-western Nigeria. Research Journal of Agricultural and Environmental Management. 2(6): 176182.

Bello. H. S, Isa. T, Isa. M. A. and Akinmuisere. K. 2013. Effects of land use on the nature and population of microorganisms in the semi-arid region of north-eastern Nigeria. International journal of environment. 2(1): 224-230

Dinesh. K, Upadhyay. G.P, Anil. D and Bhutia. K.G. 2017. Assessment of soil biological properties under different land uses in barog-dhillon watershed in solan district of Himachal Pradesh. International Journal of Chemical Studies. 5(4): 221-224

Gomez and Gomez, 1984. Statistical methods for Agricultural Research. Wiley and Wiley Publications.

Ibekwe, A.M., Poss, J.A., Grattan, S.R., Grieve, C.M., and Suarez, D., 2012. Bacterial diversity in cucumber (Cucumis sativus) rhizosphere in response to salinity, soil $\mathrm{pH}$, and boron. Soil Biology and Biochemistry. 42. 567575.

Maqbool. M, Rasool. R and Ramzan. S. (2017). Soil physico-chemical properties as impacted by different land use systems in district Ganderbal, Jammu and Kashmir: India. International Journal of Chemical Studies. 5(4): 832-840.

Michel. K.Y, Pascal K.T.A, Souleymane K, Jerome E.T, Yao T., Luc. A and Danielle. B. 2010. Effects of land-use types on soil organic carbon and nitrogen dynamics in mid-west Cote d'Ivoire. European Journal of Scientific Research.2 211-222.

Nisar, M and Lone, F.A. 2013. Effect of landuse/landcover change on soils of a kashmir himalayan catchment-sindh. International Journal of Research In Earth \& Environmental Sciences. 1(1): 13-27.

Okonkwo, C.I. (2010). Effect of Burning and Cultivation on Soil Properties and Microbial Population of Four Different Land Use Systems in Abakaliki. Research Journal of Agriculture and Biological Sciences.6 (6): 1007-1014.

Sethi, S., Mathur. N and Bhatnagar. P 2013. Effects of synthetic pyrethroids on phosphate solubilizing activity of microorganisms. International Journal of Current Microbiology and Applied Sciences. 2(12): 240-246

Sofi. J.A., Bhat. A.G., Kirmani. N.A., Wani. J.A., Lone. A.L., Mumtaz A. G., Dar. G.I.H. 2016. Soil quality index as affected by different cropping systems in northwestern Himalayas. Environment Monitoring Assessment.188:161.

Srinivasulu and Rangaswamy. 2014. OMICS Group: e Books: Enzymes and Pesticides.

Sui, X., Feng, F., Lou, X., Zheng, J., and Han, S., 2012. Relationship between microbial community and soil properties during natural succession of forest land. African Journal of Microbiology Research. 6(42): 70287034.

\section{How to cite this article:}

Fozia Shafiq Wani, Farida Akhter, Shakeel Mir, Zahoor Ahmed Baba, Showkat Maqbool, Mohammad Yousuf Zargar and Sajad Un Nabi. 2018. Assessment of Soil Microbial Status under Different Land Use Systems in North Western Zone of Kashmir. Int.J.Curr.Microbiol.App.Sci. 7(08): 266-279. doi: https://doi.org/10.20546/ijcmas.2018.708.032 